\title{
Fertilización nitrogenada en trigo para la producción de forraje y grano en el sudoeste de la provincia de Buenos Aires (Argentina)
}

\author{
Arzadun, M.; H. Laborde y J. Arroquy
}

\begin{abstract}
RESUMEN
Cuando del cultivo de trigo (Triticum aestivum) se obtiene forraje y grano, i.e. cultivo doble propósito (DP), la disponibilidad de nitrógeno puede ser un aspecto clave para la expansión de esta alternativa. Se comparó la respuesta al $\mathrm{N}$ en trigo DP fertilizado en abril (DPF), en agosto (DPG), en ambas fechas (DPFG) y en el cultivo para grano (TC) en un experimento factorial con las dosis de N: 0, 40, 80, 120, y $160 \mathrm{~kg} \mathrm{ha}^{-1}$, aplicado como urea al voleo. Se determinó el rendimiento de forraje y de grano y el contenido de $\mathrm{N}$ en cada producto. El rendimiento de forraje se incrementó en promedio en 10,3 $\mathrm{kg} \mathrm{MS} \mathrm{kg}^{-1}$ de N aplicado, hasta la dosis de $120 \mathrm{~kg} \mathrm{ha}^{-1}$. El rendimiento de grano fue menor en los DP, pero tuvo una mayor respuesta a $N$, incrementándose en 8,3, 7,3, 4,7, y $4,7 \mathrm{~kg} \mathrm{~kg}^{-1}$ de N aplicado para DPG, DPFG, DPF y TC respectivamente. La eficiencia agronómica de la respuesta a $\mathrm{N}$ en el rendimiento de grano fue superior en los manejos de DP y estos resultados muestran una mayor conveniencia de la fertilización nitrogenada en estos que en TC.
\end{abstract}

Palabras clave: nitrógeno, trigo, forraje, grano, eficiencia de respuesta.

Arzadun, M.; H. Laborde and J. Arroquy, 2013. Nitrogen fertilization of wheat for forage and grain production in the south west of Buenos Aires province (Argentina). Agriscientia 30 (1): 1-11

\section{SUMMARY}

When forage and grain are obtained from a wheat crop, (Triticum aestivum), i.e. a dual purpose crop (DP), the availability of $\mathrm{N}$ can be a key factor for the expansion of this alternative system. The response to nitrogen fertilization of a DP crop fertilized in April (DPF), in August (DPG), in both dates (DPFG) and in wheat grown only for grain (TC) was compared in a factorial experiment with the $\mathrm{N}$ levels 0, 40, 80, 120, and $160 \mathrm{~N} \mathrm{~kg} \mathrm{ha-1}^{-1}$, applied as broadcast urea. Forage and grain yield and $\mathrm{N}$ content of each product were determined. Forage yield increased, on average, $10.3 \mathrm{~kg}$ dry matter per $\mathrm{kg}$ of applied N, up to the 120 $\mathrm{kg} \mathrm{ha}^{-1}$ level. Grain yield was lower in DP than TC but had a higher response to $\mathrm{N}$, increasing on average 8.3, 7.3, 4.7, and $4.7 \mathrm{Kg}$ per $\mathrm{kg}$ applied $\mathrm{N}$, for DPG, DPFG, DPF and TC respectively. The agronomic efficiency of the response to $\mathrm{N}$ in grain yield was higher in DP than in TC and these results show that $N$ 
fertilization in DP is more convenient than in TC.

Key words: nitrogen, wheat, forage, grain, response efficiency..

M. Arzadun: Chacra Experimental Coronel Suárez, MAA Pcia. de Buenos Aires. CC 204, 7540 Coronel Suárez, Buenos Aires, Argentina. H. Laborde: Departamento de Agronomía, Universidad Nacional del Sur. Altos del Palihue, 8000 Bahía Blanca, Argentina. J. Arroquy: Comisión de Investigaciones Científicas de la Provincia de Buenos Aires. Correspondencia a: marzadun@infovia.com.ar

\section{INTRODUCCIÓN}

La experiencia proveniente de otras regiones del mundo muestra la conveniencia del trigo como cultivo de doble propósito, capaz de sostener una elevada producción animal durante el pastoreo invernal y ser luego destinado a cosecha de grano (Redmon et al.,1995). Esto coincide con los resultados de trabajos desarrollados en la región subhúmeda pampeana, que indican la factibilidad de la práctica (Hernández, 1969; Peralta et al., 2011) y que la reducción de los ingresos por la producción de grano motivada por el pastoreo es superada por el valor del producto animal logrado (Arzadun et al., 2003).

Experimentos recientes realizados en red proveen evidencia de la posible conveniencia de esta práctica en distintas regiones de la llanura pampeana (Bainotti et al., 2008) usando un número importante de las variedades de trigo comercializadas actualmente. Sin embargo, un aspecto que puede limitar el uso como doble propósito de los cereales de invierno es que la disponibilidad de nitrógeno deba ser alta al extraer del cultivo el forraje previamente a la cosecha de grano.

La producción de cereales origina el 60\% de la demanda mundial de fertilizantes nitrogenados y de estos sólo un 33\% es recuperado en los granos cosechados (Raun \& Johnson, 1999), aunque valores mucho menores pueden ser esperables. Los cultivos de producción de forraje tienen en general un uso del $\mathrm{N}$ más eficiente que los dedicados a la producción de grano (Thomason et al., 2000). Una de las razones de las diferencias en eficiencia de uso del nutriente es la existencia de pérdidas de $\mathrm{N}$ gaseosas importantes cuando el cultivo se aproxima a la floración (Raun \& Johnson, 1999).

Se ha informado que en trigos de invierno el control de la dosis y el momento de aplicación del
N mejoran la eficiencia de utilización. Así, la fertilización otoñal ajustada al N disponible en el lote, combinada con una fertilización a fin del macollaje, resulta en una mayor eficiencia, estimada como recuperación del N en grano (Sowers et al., 1994). Existe coincidencia, además, en que la eficiencia de absorción de $\mathrm{N}$ por el cultivo y su destino a producto (forraje o grano) tiende a incrementarse en la medida que su aplicación ocurra inmediatamente antes de un período de rápida absorción y crecimiento (Johnston \& Fowler, 1991). En consecuencia, muy probablemente la provisión de agua y la temperatura en el momento de fertilización tendrán una influencia tan importante como el estado de desarrollo del cultivo.

El momento de aplicación de la fertilización nitrogenada podría orientar la respuesta hacia la producción de forraje o la de grano. No existe información experimental que compare la respuesta combinada en forraje y grano al nitrógeno aplicado temprano, con la de una aplicación de fines del invierno, exclusivamente para la producción de grano.

Los incrementos en la eficiencia de uso del nutriente tienen importantes implicancias económicas y ambientales, y mediciones de la eficiencia de uso del nutriente han merecido particular atención; se han elaborado índices de diferente complejidad tomando en cuenta distintos componentes o etapas de la eficiencia (Huggins \& Pan, 1993). Un enfoque más simple para evaluar la eficiencia de la fertilización resulta el cálculo del incremento de rendimiento logrado (diferencia entre testigo y fertilizado) por unidad de nutriente aplicado. Ese valor de eficiencia agronómica (EA) (Doberman, 2007) se calcula como:

$E A=\left(R_{n}-R_{0}\right) / F_{n}$,

siendo $R_{n}$ el rendimiento obtenido con la dosis $n$, $\mathrm{R}_{0}$ su correspondiente testigo sin fertilizante, y $F_{n}$ el 
nutriente aplicado.

La calidad del forraje de trigo puede ser modificada por la fertilización nitrogenada. Una elevada provisión de nitrógeno ha resultado, en diferentes gramíneas, en cambios en la composición del forraje que pueden resultar finalmente limitantes de la producción animal. Así, un aumento del contenido de proteína bruta (PB) suele ir acompañado por una elevada solubilidad del nitrógeno dado por una proporción elevada de proteínas de bajo peso molecular, aminoácidos libres e incluso nitrógeno inorgánico (Raun \& Westerman, 1991). El nivel de carbohidratos solubles, considerado un importante indicador del valor nutritivo del forraje de gramíneas anuales de invierno, tiende a decaer con la provisión de nitrógeno (Fernando \& Carter, 1970). La evolución de estos dos componentes del forraje con el agregado de nitrógeno muestra que la fertilización nitrogenada muy probablemente no mejore la calidad del forraje, e incluso puede actuar disminuyéndola.

El impacto sobre la calidad del grano, sin embargo, es muy apreciado ya que los aumentos en el nivel proteico se relacionan con la fertilización y dan al grano una mayor calidad industrial.

El trigo DP ofrece una alternativa beneficiosa a los sistemas mixtos del sudoeste bonaerense, reemplazando parcialmente el cultivo dominante para cosecha de grano, y la fertilización nitrogenada debería adaptarse a esta modalidad productiva. La fertilización nitrogenada del trigo DP conducirá a una mayor eficiencia de uso del nutriente que la del cultivo manejado solamente para producir grano.

El objetivo de este trabajo fue comparar el resultado de la fertilización nitrogenada para la producción de forraje y de grano de trigo con la dirigida a incrementar el rendimiento de grano sobre el cultivo de trigo en el sudoeste sub-húmedo de Buenos Aires, Argentina.

\section{MATERIALES Y MÉTODOS}

El experimento se realizó en dos localidades del sudoeste de la provincia de Buenos Aires (Argentina): Pasman ( $\left.38^{\circ} 21^{\prime} \mathrm{S}, 62^{\circ} 08^{\prime} \mathrm{O}\right)$, en los años 1998 y 2000 (experimentos P98 y P00), y Cabildo

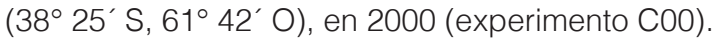
El suelo de Pasman es un Hapludol petrocalcico de textura franca con presencia de tosca a unos 60-75 cm de profundidad. El suelo de Cabildo fue clasificado como un Hapludol típico de textura franco arenosa con el horizonte petrocálcico no detectado en los primeros $110 \mathrm{~cm}$.
Un análisis del suelo de los primeros $20 \mathrm{~cm}$, en cada sitio experimental, practicado en marzo mostró contenidos de fósforo asimilable de 9, 12 y 10 P $\mathrm{mg} \mathrm{kg}^{-1}$ (método Bray y Kurtz), de materia orgánica de 44, 21 y $48 \mathrm{~g} \mathrm{~kg}^{-1}$ (método Walkley y Black) y de nitrógeno como nitratos $\left(\mathrm{NO}_{3}-\mathrm{N}\right)$ de 6,0, 2,2 y 4,9 $\mathrm{mg} \mathrm{kg}^{-1}$, para P98, C00 y P00, respectivamente. En el momento de la siembra se aplicaron $45 \mathrm{~kg}$ ha $^{-1}$ de fosfato di-amónico incorporado con rastra de discos al suelo.

Un muestreo de suelo $(0-20 \mathrm{~cm})$ sobre las parcelas de trigo doble propósito y de cosecha de grano que no recibieron fertilización con urea, fue practicado en abril (luego de la implantación del trigo doble propósito), en junio (cuando se sembró el cultivo para cosecha de grano) y a fines de agosto (último aprovechamiento del doble propósito y comienzo de encañazón). Se estimó de este modo la evolución del contenido de nitratos, extraídos con $\mathrm{KCl}$ y medidos por colorimetría (Keeney \& Nelson, 1982).

\section{Diseño experimental}

En un experimento factorial en parcelas divididas sobre cuatro bloques completos al azar se distribuyeron, como parcelas principales, cuatro tratamientos consistentes en manejos del cultivo de trigo cv Prointa Super, tres de doble propósito sembrados en la segunda quincena de marzo y uno para la cosecha de grano sembrado a mediados de junio. La densidad de siembra fue, en todos los casos, de 250 semillas viables $\mathrm{m}^{-2}$.

La labranza fue convencional, comenzando la preparación del suelo a fin de febrero con rastra de doble acción. El área destinada a las parcelas de siembra más tardía (TC) fue mantenida libre de malezas mediante herbicida glifosato (sal isopropilamina del ácido $\mathrm{N}$-[fosfonometil] glicina); se tuvo en este caso un barbecho de unos 120 días a la siembra.

Los tratamientos de doble propósito se diferenciaron por el momento de aplicación del nitrógeno, según fuera dirigido a la producción de forraje (mediados de abril) (DPF), grano (agosto) (DPG) o fraccionado en ambos momentos (DPFG). Ambas épocas de fertilización fueron aproximadamente coincidentes con el comienzo y la finalización del macollaje. El trigo para cosecha de grano (TC) recibió el nitrógeno en igual momento que DPG.

Dentro de cada manejo fueron distribuidos como parcela secundaria (área de 1,5 x $7 \mathrm{~m}$ ) cinco niveles de fertilización correspondientes a las dosis de: 0 (testigo), 40, 80, 120 y 160 kg de nitrógeno por 
hectárea. La aplicación de nitrógeno fue realizada, en todos los casos, como urea al voleo.

\section{Rendimiento de forraje y de grano}

La producción de forraje fue evaluada mediante cortes con moto-guadañadora sobre un área central de cada parcela de $5 \mathrm{~m}^{2}$, realizados a una altura de 3-5 cm, en dos momentos: en la segunda quincena de junio (evaluando la acumulación de forraje otoñal) y cuando el cultivo comenzaba el encañado y resultaban visibles los primeros entrenudos huecos (fin de agosto). Una muestra del material cortado se secó en estufa a $65^{\circ} \mathrm{C}$, para estimar contenido de materia seca y realizar posteriores análisis químicos.

Al alcanzar el cultivo el estado de madurez comercial se cortó la planta entera sobre el área central de $5 \mathrm{~m}^{2}$ de cada parcela, se hizo la trilla y se determinó el peso del grano obtenido y se corrigió el rendimiento a una humedad de $12 \%$. Muestras del grano fueron secadas en estufa y molidas para su análisis.

La eficiencia agronómica de respuesta a la fertilización se calculó como el cociente entre el incremento de rendimiento, de forraje o de grano, obtenido dividido por la dosis de $\mathrm{N}$ aplicada. Dicho incremento de rendimiento fue la diferencia entre el de parcelas fertilizadas y su correspondiente testigo $\sin \mathrm{N}$, dentro de cada manejo y repetición; en el caso del forraje sólo se tomó en cuenta el manejo DPF.

Sobre las muestras de forraje y de grano de los tratamientos PDF, PDG y TC se realizaron estimaciones del $\mathrm{N}$ total usando el procedimiento Kjeldhal (AOAC, 1990). El contenido de proteína bruta $(P B)(N \times 6,25)$, fibra detergente neutro y fibra detergente ácido (Goering \& Van Soest, 1970), carbohidratos solubles (Thomas, 1977) y lignina fueron determinados como parámetros de calidad del forraje. El contenido proteico del grano también fue calculado como PB $(\mathrm{N} \times 5,75)$. Todas las determinaciones fueron realizadas en el Laboratorio de Nutrición Animal del Departamento de Agronomía de la Universidad Nacional del Sur.

\section{Análisis estadístico}

Los resultados obtenidos fueron sometidos a análisis de varianza para el correspondiente modelo de parcelas divididas, combinado sobre los tres experimentos, usando contrastes ortogonales para evaluar las tendencias de respuesta al $\mathrm{N}$ y la prueba de comparaciones múltiples (LSD) para contrastar medias de los diferentes manejos. Las regresiones obtenidas para la respuesta a la fertilización, en forraje o en grano, fueron analizadas para la significancia estadística de sus componentes lineales y cuadráticos.

\section{RESULTADOSY DISCUSIÓN}

\section{Clima}

Si bien en los tres experimentos la humedad del suelo fue suficiente para la implantación en ambas fechas de siembra, el trabajo abarcó un período de precipitaciones bajas en relación al promedio de 33 años en cada localidad (Tabla 1). El período invernal, de junio a agosto fue particularmente seco en todos los casos y las lluvias primaverales resultaron muy escasas en $\mathrm{P} 98$ respecto al promedio de Pasman.

\section{Nitratos en el suelo}

La Tabla 2 muestra la evolución del contenido de nitrógeno como nitratos $\left(\mathrm{N}-\mathrm{NO}_{3}\right)$ en el suelo de las parcelas sin fertilización nitrogenada (testigos).

Tabla 1. Precipitaciones mensuales registradas durante el trabajo y promedio de 33 años (1976 a 2008) en Pasman y en Cabildo.

\begin{tabular}{|c|c|c|c|c|c|}
\hline Mes & \multicolumn{3}{|c|}{ Periodo experimental } & \multicolumn{2}{|c|}{ Promedio de 33 años } \\
\hline & P98 & $\mathrm{POO}$ & $\mathrm{COO}$ & Pasman & Cabildo \\
\hline Enero & 144 & 83 & 50 & 103,2 & 70,1 \\
\hline Febrero & 240 & 132 & 12 & 94,7 & 67,1 \\
\hline Marzo & 27 & 127 & 31 & 110,8 & 72,2 \\
\hline Abril & 113 & 14 & 9 & 72,7 & 60,8 \\
\hline Mayo & 30 & 73 & 74 & 47,6 & 37,6 \\
\hline Junio & 0 & 0 & 16 & 20,0 & 23,5 \\
\hline Julio & 17 & 0 & 21 & 28,7 & 27,7 \\
\hline Agosto & 28 & 52 & 25 & 33,5 & 35,9 \\
\hline Septiembre & 102 & 62 & 51 & 57,8 & 45,6 \\
\hline Octubre & 35 & 178 & 70 & 97,9 & 73,5 \\
\hline Noviembre & 66 & 15 & 25 & 96,5 & 59,7 \\
\hline Diciembre & 36 & 37 & 10 & 86,5 & 67,7 \\
\hline
\end{tabular}


Tabla 2. Contenido de nitrógeno como nitratos (N-NO3 \%) en el suelo $(0-20 \mathrm{~cm})$ en tres momentos en cada experimento y en diferente manejo, en parcelas sin fertilización nitrogenada.

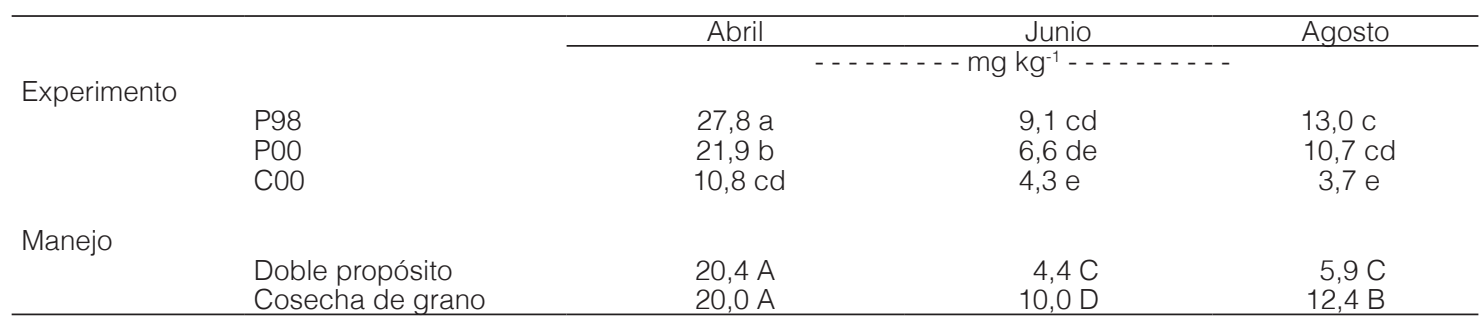

Medias seguidas de igual letra minúscula, dentro de experimento $x$ momento, o mayúscula dentro de manejo $x$ momento, no se diferencian según los respectivos valores de $\operatorname{LSD}(P \leq 0,05)$.

Estos niveles de nitratos variaron según la fecha de muestreo, el experimento y el manejo ( $P \leq 0,01)$, con interacción significativa para experimento $\mathrm{x}$ fecha y manejo $x$ fecha $(P \leq 0,05)$.

Los niveles de $\mathrm{N}-\mathrm{NO}_{3}$ resultaron siempre mayores en P98 y sensiblemente menores en C00 y en todos los casos de observó una importante reducción de abril a junio. A partir de similares niveles en abril el trigo doble propósito mostró valores inferiores que el de cosecha de grano en los muestreos posteriores. Aún así, en el trigo de cosecha de grano los nitratos se redujeron a la mitad de abril a junio, mientras esas parcelas permanecían en barbecho. Esto resultó coincidente en los tres experimentos y pudo ser causado fundamentalmente por la inmovilización de ese $\mathrm{N}$ en la materia orgánica del suelo; en las condiciones de humedad de este trabajo puede descartarse una significativa pérdida por lixiviación (Lazzari, 1992).

En agosto, cuando el doble propósito recibió su último corte y comenzó la encañazón, estas diferencias se mantuvieron o se acrecentaron, especialmente en $\mathrm{PO0}$, donde el nivel de nitratos de TC casi quintuplicó el nivel del de doble propósito. Estas diferencias pueden ser interpretadas a partir de condiciones climáticas particularmente favorables en P00 para la mineralización del N que luego sustentaron una elevada producción de grano y al mismo tiempo una baja respuesta a la fertilización en TC en ese experimento, como se verá más adelante.

\section{Rendimiento de forraje}

Los dos cortes realizados para evaluar el rendimiento de forraje ocurrieron entre el 26 y 28 de junio, para el rendimiento otoñal, y entre el 12 y 25 de agosto, para el invernal, en los tres experimentos. Tanto la acumulación de forraje como la respuesta al nitrógeno estuvieron repartidas en magnitudes aproximadamente similares entre ambos períodos
$(P>0,25)$ en los tres experimentos y esto hace que se analice el rendimiento total como suma de ambos cortes.

El rendimiento total de forraje (Figura 1) evolucionó desde niveles cercanos a los $900 \mathrm{~kg}$ de MS/ ha hasta un máximo superior a las 4 toneladas, con diferencias significativas atribuibles a experimento, nivel de nitrógeno y su interacción ( $P \leq 0,01)$. Las comparaciones realizadas entre los experimentos muestran que la producción obtenida en los de la localidad Pasman fue superior a la del experimento de Cabildo (CO0).

Mientras la respuesta en P98 fue lineal la de los experimentos P00 y C00 admiten un componente cuadrático $(P=0,001)$ determinado por el nivel más alto de fertilización (160 Kg N ha ${ }^{-1}$ ). La respuesta lineal obtenida en los tres experimentos hasta la dosis de $120 \mathrm{~kg} \mathrm{~N}$ ha- (Fig.1) representó un incremento de 13,37, 9,19 y 8,23 kg de MS por kg de $\mathrm{N}$ aplicado en P98, C00 y P00, respectivamente, sin alcanzar diferencias significativas entre los experimentos. La ordenada al origen (representa el cultivo sin fertilización) fue estadísticamente diferente en los tres experimentos ( $P \leq 0,01)$, con valores de 2010, 904, y $2929 \mathrm{~kg}$ de $\mathrm{MS} \mathrm{ha}^{-1}$, res-

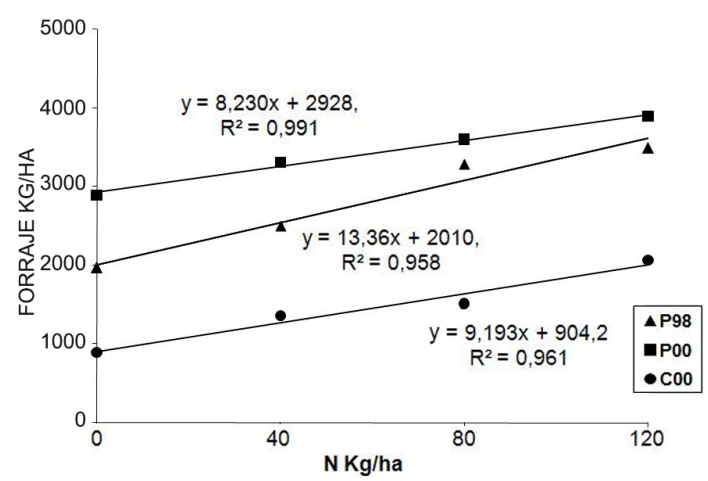

Figura 1. Respuesta del rendimiento de forraje de trigo al nitrógeno en Pasman (P98 y P00) y Cabildo (C00) entre 0 y 120 $\mathrm{Kg}$ de $\mathrm{N} \mathrm{ha}^{-1}$, y su ajuste lineal. 
pectivamente para P98, C00 y P00 (LSD $0,05=746)$. El menor rendimiento general de forraje obtenido en C00 se debe relacionar con el menor nivel de materia orgánica observado inicialmente en el suelo de ese experimento.

\section{Rendimiento de grano}

El rendimiento de grano varió desde valores cercanos a los $1000 \mathrm{~kg} \mathrm{ha}^{-1}$ hasta los $5000 \mathrm{~kg} \mathrm{ha}^{-1}$ en función de las distintas opciones de manejo del cultivo y nivel de fertilización $(P \leq 0,01)$ y esas diferencias tomaron diferente magnitud en cada experimento pero manteniendo similares tendencias en la respuesta al nitrógeno.

Una significativa interacción manejo $x$ experimento $(P \leq 0,01)$ (Figura 2) puede relacionarse con el clima del periodo y las características del suelo en cada caso. Se destacan los rendimientos relativamente elevados de grano obtenidos en P00 que tienen relación con una mayor provisión de agua en ese experimento, particularmente entre agosto y noviembre (306 mm), que elevó el rendimiento marcadamente en TC. En los otros dos experimentos (P98 y C00), donde las condiciones climáticas no fueron tan favorables, entre comienzos de encañazón y antesis las diferencias en rendimiento promedio de grano entre el cultivo de doble propósito y TC se redujeron.

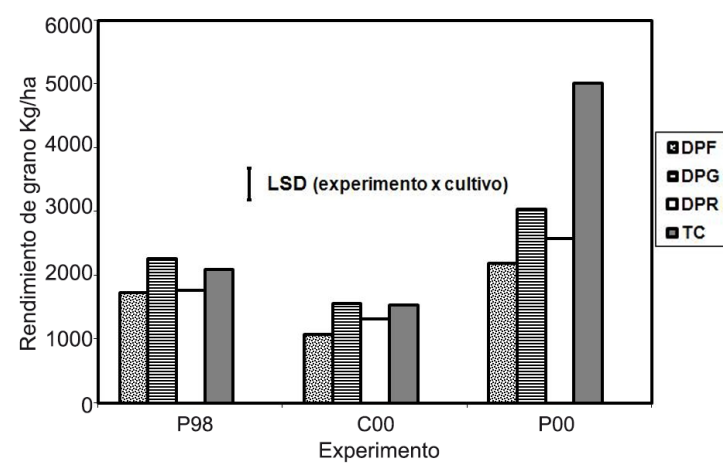

Figura 2. Rendimiento de grano de trigo en tres experimentos (promedio de distintas dosis de nitrógeno), para distintas opciones de manejo del cultivo (DPF: doble propósito fertilización temprana, DPG: doble propósito fertilización tardía, DPR: doble propósito fertilización repartida, y TC: trigo cosecha).

La respuesta a la fertilización fue particular de cada manejo, coherentemente en los tres experimentos, y tuvo un ajuste lineal por regresión, significativo y diferente en cada caso (Figura 3), sin efecto significativo del componente cuadrático. De este modo, el rendimiento de grano fue incre-

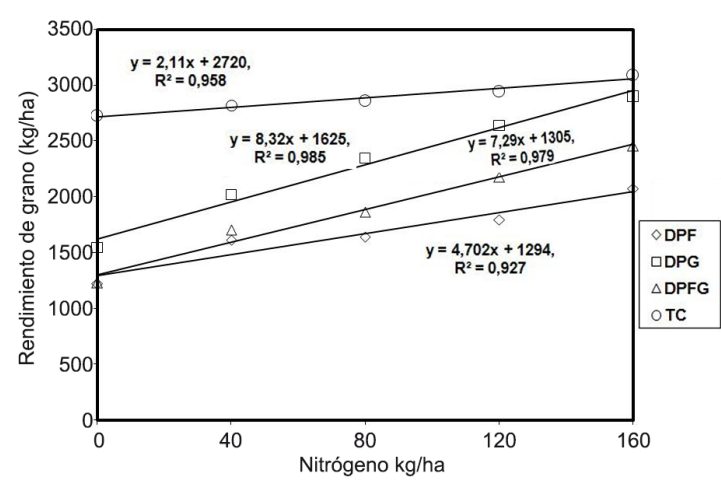

Figura 3. Respuesta al nitrógeno en el rendimiento de grano de trigo, según manejo (DPF:doble propósito fertilización temprana, DPG: doble propósito fertilización tardía, DPFG: doble propósito fertilización dividida, y TC: trigo cosecha), promedio de tres experimentos.

mentado en $4,70,8,32,7,29$ y $4,71 \mathrm{~kg}$ de grano por kg de $\mathrm{N}$ aplicado, para TC, DPG, DPFG y DPF, respectivamente. En promedio, los rendimientos de grano a la dosis de $\mathrm{N}$ más elevada resultan similares $(P>0,10)$ para DPG y TC, aunque el rendimiento obtenido en ausencia de fertilización fue siempre mayor en TC.

La baja respuesta en TC se puede atribuir a escasez de agua en el cultivo en primavera temprana en C00 y a una elevada provisión de $\mathrm{N}$ en el suelo en similar período en P98 y P00, según muestra el nivel de nitratos en el suelo en agosto en P98 y P0O (Tabla 2). Ambos factores, la sequía y el $\mathrm{N}$ disponible en el suelo redujeron la respuesta en TC que en las parcelas sin fertilización mantuvo un rendimiento relativamente alto (el más elevado fue de 3,9 toneladas/ha en P00) para las condiciones climáticas de este trabajo.

\section{Eficiencia agronómica de uso del nitrógeno}

La eficiencia agronómica de la respuesta al nitrógeno aplicado, calculada para los incrementos de rendimiento de forraje, fue distinta entre experimentos y tendió a caer linealmente con el incremento de la dosis $(P \leq 0,10)$ sin interacción significativa entre ambos factores (Tabla 3 ). En general, esta eficiencia resulta relativamente baja respecto a la encontrada en diferentes trabajos con cultivos forrajeros anuales de invierno en condiciones similares. El hecho de considerar sólo la acumulación de forraje otoño-invernal del cultivo limitó la respuesta obtenida. En los mismos sitios de este trabajo, en un experimento se aplicó urea en abril sobre trigo, avena, cebada, raigrás anual, y triticale, y se originaron niveles de eficiencia agronómica en la respuesta en forraje superiores a $10 \mathrm{~kg} \mathrm{MS}$ 
$\mathrm{kg}^{-1}$ de $\mathrm{N}$ aplicado en $75 \%$ de los casos, cuando el rendimiento primaveral de forraje fue considerado, pero iguales o inferiores a los de éste trabajo si se computaba sólo el rendimiento otoño invernal (Freddi et al, 2003). Una mayor eficiencia de respuesta observada en P98 se puede relacionar con las mayores lluvias otoñales de ese experimento.

La eficiencia agronómica de la respuesta en grano manifestó efectos significativos de experimento, nivel de $\mathrm{N}$ y manejo, sin interacciones significativas entre estos factores (Tabla 3). Los tres experimentos mostraron niveles medios diferentes relacionados con las Iluvias ocurridas en la primavera, en cada caso. (Tabla 1 y Tabla 3). De igual manera que para el forraje, la eficiencia agronómica del grano manifiesta una tendencia lineal decreciente con el aumento de la dosis de fertilizante. En todos los casos, la mayor caída de eficiencia se observó luego de la dosis de 40 kg ha-1; trabajos realizados en la región sostienen que la mayor eficiencia se daría en niveles de fertilización similares a este (Loewy, 1990).

La totalidad de los valores de eficiencia agronómica de respuesta en grano encontrados en este trabajo están comprendidos en el amplio rango reportado por Loewy (1990) para cultivos de trigo de esta misma región del sudoeste bonaerense. Lo más notable es la diferencia observada entre los diferentes manejos, especialmente entre DPG y $\mathrm{TC}$, tratamientos que recibieron la fertilización en el mismo momento. Un contraste ortogonal adicional entre los manejos de doble propósito y TC muestra la superior eficiencia de los primeros $(P \leq 0,05)$.

El $\mathrm{N}$ exportado como forraje, como grano y en total de las parcelas testigo sin fertilizante mostró una diferencia entre los experimentos $(P \leq 0,10)$ similar a la de los rendimientos ya presentados. No existieron interacciones significativas entre los experimentos y los manejos en el nitrógeno exportado de las parcelas sin fertilización, con $80 \mathrm{~kg} \mathrm{ha}^{-1}$, ni en la diferencia entre ambos tratamientos ( $\mathrm{N}$ de la respuesta al fertilizante).

En el testigo sin fertilizante, el total de $\mathrm{N}$ exportado en los manejos de DP (Tabla 4) resultó superior al de TC debido al forraje obtenido de ellas (aproximadamente $60 \%$ del total). La fertilización aplicada en cada manejo cambió las proporciones de $\mathrm{N}$ exportado, incrementando la fracción del forraje en DPF y la del grano en DPG. El total exportado en productos, así como el $\mathrm{N}$ exportado en la respuesta a la fertilización, muestran un mayor nivel en DPF, intermedio en DPG y más bajo en TC.

El $N$ exportado en la respuesta total al fertilizante en relación a la dosis aplicada fue de 54,1\% para DPF (forraje y grano), de $22,7 \%$ para DPG y de $10,6 \%$ para TC. Los niveles de DPG y TC (correspondiente a sólo grano) resultan cercanos a los niveles de recuperación de $\mathrm{N}^{15}$ reportados en experimentos de la región (Laurent et al., 1996) o con los resultados de una serie de evaluaciones de la eficiencia de uso del N (Raun et al., 2002).

La respuesta obtenida en forraje origina un aprovechamiento mayor del $\mathrm{N}$ que la de grano, coincidente con la información previa (Thomason et al., 2000). De todos modos, esto no implica necesariamente una conveniencia económica debido a la transformación de forraje en productos ganaderos y a las variables relaciones de ingreso neto que

Tabla 3. Eficiencia agronómica de la respuesta al fertilizante nitrogenado en forraje y en grano, para los tres experimentos, según dosis de nitrógeno aplicado, y en grano para los diferentes manejos.

\begin{tabular}{|c|c|c|}
\hline & $\begin{array}{c}\text { Forraje } \\
\mathrm{Kg} \mathrm{MS} \mathrm{kg}^{-1} \mathrm{~N} \text { aplicado }\end{array}$ & $\begin{array}{c}\text { Grano }^{1} \\
\text { Kg grano kg-1 } \mathrm{N} \text { aplicado }\end{array}$ \\
\hline \multicolumn{3}{|l|}{ Experimento } \\
\hline P98 & $14,16 \mathrm{a}$ & $6,91 \mathrm{ab}$ \\
\hline POO & $8,10 \mathrm{~b}$ & $10,28 \mathrm{a}$ \\
\hline $\mathrm{COO}$ & $8,61 \mathrm{~b}$ & $5,28 \mathrm{~b}$ \\
\hline Efecto & * & * \\
\hline \multicolumn{3}{|l|}{ Dosis de $\mathrm{N}\left(\mathrm{kg} \mathrm{ha}^{-1}\right)$} \\
\hline 40 & 11,78 & 10,37 \\
\hline 80 & 11,02 & 6,72 \\
\hline 120 & 10,26 & 6,44 \\
\hline 160 & 8,10 & 6,43 \\
\hline Efecto & $*, L^{*}$ & $*, L^{*}$ \\
\hline \multicolumn{3}{|l|}{ Manejos } \\
\hline DPF & --- & $6,25 a b$ \\
\hline$D P G$ & -- & 9,86 a \\
\hline DPFG & --- & $8,82 \mathrm{a}$ \\
\hline $\begin{array}{l}\text { TC } \\
\text { Efecto }\end{array}$ & --- & $\underset{*}{5,02} b$ \\
\hline
\end{tabular}

${ }^{1}$ Grano con $12 \%$ de humedad.

*: $P \leq 0,10 ; L^{*}$ tendencia lineal $(P \leq 0,10)$

Medias de la misma columna seguidas de igual letra no difieren según LSD $(P \leq 0,05)$ 
Tabla 4. Nitrógeno exportado por parcelas de trigo sin fertilizante, por las que recibieron $80 \mathrm{~kg} \mathrm{~N} \mathrm{ha}^{-1}$, y la diferencia entre ambas, promedio de tres experimentos.

\begin{tabular}{|c|c|c|c|c|c|c|c|c|c|}
\hline & \multicolumn{3}{|c|}{ Testigo $\sin N$} & \multicolumn{3}{|c|}{ Con 80 kg $\mathrm{N}$ ha-1 } & \multicolumn{3}{|c|}{ Diferencia } \\
\hline & Forraje & Grano & Total & Forraje & Grano & Total & Forraje & Grano & Total \\
\hline DPF & 47,1 & $26,6 \mathrm{~b}$ & $73,7 \mathrm{a}$ & 85,1 & $31,9 \mathrm{c}$ & $117,0 \mathrm{a}$ & 38,0 & 5,3 & $43,3 a$ \\
\hline DPG & 46,3 & $28,4 b$ & $74,7 \mathrm{a}$ & 46,3 & $46,6 \mathrm{~b}$ & $92,9 \mathrm{~b}$ & 0 & 18,2 & $18,2 \mathrm{~b}$ \\
\hline $\mathrm{TC}$ & --- & $52,1 \mathrm{a}$ & $52,1 \mathrm{~b}$ & --- & 60,6 a & $60,6 \mathrm{c}$ & --- & 8,5 & $8,5 \mathrm{c}$ \\
\hline Anova $^{1}$ & NS & ** & ** & * & ** & ** & --- & NS & ** \\
\hline
\end{tabular}

1 Efecto de manejo en el análisis de varianza

NS: $\mathrm{P}>0,10 ;{ }^{*}: \mathrm{P} \leq 0,10 ;{ }^{* *}: \mathrm{P} \leq 0,01$

Medias de la misma columna seguidas de igual letra no defieren según LSD $(P \leq 0,05)$.

estos productos y la venta del grano generan.

En ambientes más húmedos que los de este trabajo y con suelos arenosos, pueden producirse pérdidas importantes por lixiviación, especialmente ante el uso de dosis elevadas de $\mathrm{N}$ en fecha temprana. En esas circunstancias el fraccionamiento de la aplicación del fertilizante, como en DPFG puede minimizar las pérdidas aumentando la eficiencia de uso (Loewy, 2004). En las condiciones de este trabajo, con respuesta lineal hasta dosis relativamente elevadas, el fraccionamiento de la dosis no condujo a mejorar el uso del nutriente para la producción de grano.

\section{Efectos sobre la calidad del forraje}

La calidad del forraje de trigo obtenido en estos experimentos se puede considerar elevada debido a su contenido promedio de PB, carbohidratos solubles y fibras. En todos los parámetros considerados existieron diferencias atribuibles al experimento y al corte (temprano o tardío) no expuestas aquí (Denda et al, 2004), mientras el nitrógeno agregado sólo modificó significativamente $(P \leq 0,01)$ los niveles de PB y la interacción entre nivel de fertilización y el experimento no resultó significativa en ningún parámetro.

La Tabla 5 muestra un aumento lineal del nivel proteico del forraje con el aumento de la dosis de $\mathrm{N}$. El rango de valores observado se encuentra en todos los casos por encima de los requerimientos de rumiantes en engorde y aun supera, en general, a los de vacas de alta producción lechera. Estos niveles de PB calculados, por lo tanto, no implicarán necesariamente un aumento de la calidad del forraje per se, pero indican una posible complementariedad de este forraje con otros de nivel proteico menor (NRC, 1980).

La fertilización temprana logra una mayor acumulación de forraje durante el otoño pero al mismo tiempo eleva los niveles de $\mathrm{N}$ contenido en un forraje normalmente rico en $\mathrm{PB}$. Este fenómeno, ade-
Tabla 5. Niveles de proteína y carbohidratos hidrosolubles en el forraje de trigo según la dosis de $\mathrm{N}$ aplicado como urea. Promedio de experimentos y fechas de corte.

\begin{tabular}{|c|c|c|}
\hline Dosis & Proteína cruda & $\begin{array}{c}\text { Carbohidratos } \\
\text { solubles }\end{array}$ \\
\hline $\mathrm{N} \mathrm{Kg} \mathrm{ha}^{-1}$ & $-\cdots-\cdots-g$ & ${ }^{1} \mathrm{MS} \ldots \ldots$ \\
\hline 0 & 158 & 273 \\
\hline 40 & 166 & 256 \\
\hline 80 & 177 & 260 \\
\hline 120 & 189 & 250 \\
\hline 160 & 203 & 239 \\
\hline Efecto de $\mathrm{N}$ & $* *$ & NS \\
\hline $\begin{array}{l}\text { Tendencia } \\
\text { lineal }\end{array}$ & $\star *$ & --- \\
\hline
\end{tabular}

más de implicar una elevada remoción de $\mathrm{N}$ durante el aprovechamiento forrajero puede representar un factor indeseable desde el punto de vista de la nutrición animal (MacKown \& Carver, 2005) debido a su acumulación como $\mathrm{N}$ no-proteico.

Las dificultades para proveer a bovinos de una dieta adecuada debido a una elevada solubilidad ruminal del $\mathrm{N}$ han sido detectadas con forrajes frescos conteniendo niveles de PB muy superiores a $200 \mathrm{~g} \mathrm{~kg}^{-1}$ de MS (Fernando \& Carter, 1970; Freddi et al.,1997). Los niveles de PB encontrados en este trabajo hacen inferir que el forraje de trigo, aun con elevada fertilización nitrogenada, mantuvo una composición adecuada en sus proteínas.

Un bajo nivel de carbohidratos hidrosolubles en el forraje también ha sido señalado como una característica de la respuesta a dosis elevadas de $\mathrm{N}$, como las de estos experimentos (Fernando \& Carter, 1970). Sin embargo, los niveles encontrados en este caso se mantienen elevados, aun con una leve tendencia decreciente con el agregado de $\mathrm{N}$, no significativa (Tabla 5). Pordomingo et al. (2007) muestran que el nivel de carbohidratos solubles resultó la variable independiente que explica en mayor medida las variaciones de ganancia individual de animales pastoreando verdeos de avena. Los niveles encontrados en el forraje de trigo en este caso, mayores a $200 \mathrm{~g} \mathrm{Kg}^{-1}$ MS deberían permitir ganancias individuales de peso vivo superiores a 
los $900 \mathrm{~g}$ animal $^{-1} \mathrm{dí}^{-1}$, de acuerdo a la ecuación lineal ajustada en ese trabajo.

En cuanto al contenido de FDN, FDA y lignina del forraje obtenido en este trabajo (datos no mostrados), se observó una reducción lineal de los tres parámetros con el aumento de la dosis de N. Esta reducción, aunque significativa, no representaría un cambio importante en la calidad forrajera de la materia seca obtenida y se corresponden a un alimento de bajo contenido de fibra y elevada degradabilidad ruminal (Denda et al., 2004).

\section{Proteína del grano}

Los niveles de proteína del grano fueron significativamente diferentes $(P \leq 0,01)$ para los experimentos, los manejos y los niveles de $\mathrm{N}$ agregado como urea. De las interacciones entre estos factores sólo resultó significativa la de experimento $x$ manejo $(P \leq 0,01)$. Se observa una tendencia en el nivel proteico (promedio de niveles de fertilización) que crece de DPF a DPG y a TC definida en P00, menos marcada en P98 y que no se presentó en C00 (Figura 4)

Los niveles de proteína del grano de los tres experimentos y las diferencias entre tratamientos principales observadas resultan coherentes con los niveles de $\mathrm{N}-\mathrm{NO}_{3}$ observados en el suelo en agosto (Tabla 2) y un diferente impacto de la fertilización tardía en cada experimento condicionado por las lluvias y su oportunidad.

El efecto de la fertilización nitrogenada mostró un incremento creciente con el aumento de las dosis de N (Figura 5), similar en los distintos manejos y experimentos con un contraste cuadrático significativo $(P \leq 0,01)$.

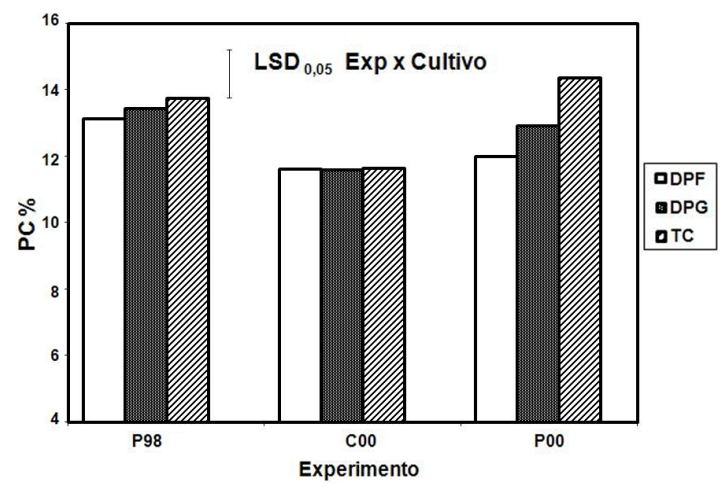

Figura 4. Nivel promedio de proteína del grano de trigo según tres diferentes manejos en tres experimentos.
El conjunto de los resultados de este trabajo muestra mayor eficiencia de uso del $\mathrm{N}$ en la producción de forraje que en la de grano, en coincidencia con la información reportada en diferentes trabajos (Raun \& Johnson, 1999; Thomason et al., 2000). La oportunidad de aplicación del nutriente, asimismo, puede modificar la relación entre los dos productos de un cultivo de doble propósito. En el presente trabajo la dosis de $40 \mathrm{~kg}$ de $\mathrm{N}$ aplicado en abril incrementó en promedio el rendimiento de forraje en aproximadamente $25 \%$, y el de grano en un $19 \%$. El mismo nivel de $\mathrm{N}$ aplicado en agosto incrementó el rendimiento de grano en un 31\%.

La conveniencia económica de la fertilización de un cultivo de doble propósito está necesariamente asociada a las relaciones entre el costo de fertilización y los precios obtenidos por grano y el producto animal (ej.: peso vivo) a vender. La conversión del forraje en producto animal puede también variar. En experimentos donde la carga animal se ajustó a la disponibilidad forrajera para mantener una elevada ganancia individual de peso, es posible observar relaciones entre el rendimiento de forraje evaluado y el peso vivo acumulado de 10$12 \mathrm{~kg}$ de MS por kg (Arzadun et al., 2003). Sin embargo, en las explotaciones existen variaciones en la asignación de forraje o limitantes a la ganancia de peso individual que pueden hacer ampliar este valor de conversión en producto animal. Por eso, se puede estimar la obtención de un kg de aumento de peso vivo por cada $20 \mathrm{~kg}$ de materia seca obtenida en la evaluación agronómica del cultivo.

Considerando los precios del trigo y del $\mathrm{kg}$ de peso vivo en el Mercado de Liniers registrados en los últimos 20 años, sus costos de comercialización y fletes, y una actualización a moneda corriente de diciembre de 2012 (AACREA, 2012) se puede estimar el ingreso neto medio obtenido por

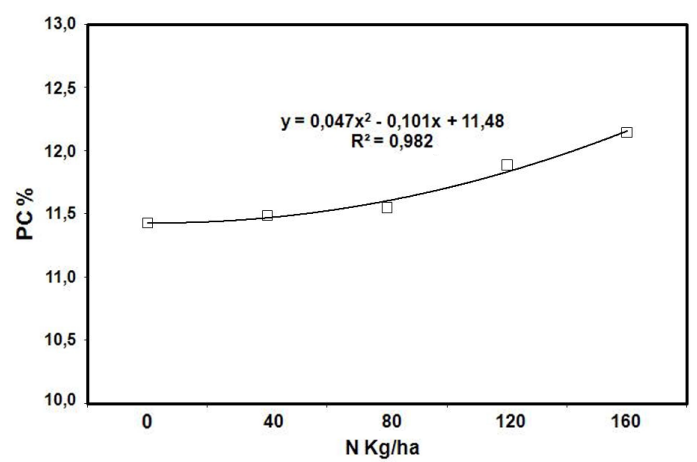

Figura 5. Incremento del contenido proteico del grano de trigo con la fertilización nitrogenada, promedio de los manejos y los experimentos. 
venta de cada producto. Si se considera del mismo modo el precio de la urea en ese lapso, su flete y el costo de aplicación para la dosis correspondiente al nivel de $40 \mathrm{~kg} \mathrm{~N} \mathrm{ha}^{-1}$, se puede calcular el retorno de la fertilización para las opciones de manejo consideradas en este trabajo a partir de la eficiencia agronómica de respuesta a dicho nivel. De este modo, se obtienen valores de 179, 179, 121 y 15 $\$$ ha $^{-1}$, para el retorno de la fertilización en DPF, DPFG, DPG y TC, respectivamente. Respecto a la inversión realizada en fertilización, esto significa un retorno de $86 \%, 86 \%, 58 \%$ y $7 \%$, respectivamente para DPF, DPFG, DPG y TC.

La conveniencia de obtener forraje adicional en respuesta a la fertilización debe ser evaluada, además, desde el punto de vista de su aporte invernal a una cadena forrajera de engorde en un momento de déficit debido a las bajas temperaturas y escasas lluvias, situación esperable en la región sudoeste bonaerense. En ese momento del año escasean los recursos forrajeros pastoriles y la alimentación invernal de los animales con granos, silajes u otros forrajes representa un costo mayor que el del forraje adicional obtenido por respuesta a la fertilización. Este forraje mantuvo características nutricionales adecuadas para la obtención de una elevada producción individual, a pesar de haber sido modificado en su composición por la fertilización.

Parte del N aplicado temprano generó una respuesta en forraje y luego una en grano de aproximadamente la mitad que la que generaría la misma dosis en aplicación de fin de macollaje. La absorción y re movilización de parte del $\mathrm{N}$ en tejidos de la planta remanentes del aprovechamiento del forraje puede contribuir a esta respuesta, así como una inmovilización en el suelo (Golik et al., 2003). Este efecto diferido en el tiempo que sería esperable bajo las restricciones hídricas de este trabajo contribuyó en este caso a que parte de la respuesta a la fertilización temprana fuera obtenida en grano.

No surge de este trabajo una particular conveniencia de la aplicación dividida del nitrógeno, sino más bien un comportamiento intermedio entre las respuestas de forraje y grano obtenidas en la aplicación temprana y la de grano correspondiente a la aplicación tardía. Se infiere de estos datos que en DPFG la respuesta en grano había estado compuesta en aproximadamente $30 \%$ por nitrógeno aplicado temprano y el resto por el más tardío. Una mayor seguridad de la respuesta en aplicaciones repartidas puede ser un factor a considerar (Loewy, 2004) ante los efectos climáticos que modifican la eficiencia de respuesta.
La inclusión de TDP remite a la planificación de los sistemas mixtos del área, y de adoptarse esta práctica demandaría más $\mathrm{N}$ que el cultivo especializado en grano, y diferentes momentos de aplicación del fertilizante. Tanto la calidad del forraje como la del grano, al respecto, son variables relevantes para la tecnología de fertilización nitrogenada. En cualquier caso, el TDP presenta menor riesgo económico y ambiental, por la mayor recuperación del $\mathrm{N}$ aplicado.

La información generada en este trabajo mostró que la fertilización del trigo para doble propósito condujo a utilizar el nutriente con mayor eficiencia que la de un cultivo para la producción de grano.

\section{AGRADECIMIENTOS.}

A Cristian Ibarra por su esmerada participación en el trabajo experimental. Al doctor Tomás Loewy por la revisión crítica del manuscrito.

\section{BIBLIOGRAFÍA}

AACREA (Asociación Argentina de Consorcios Regionales de Experimentación Agropecuaria), 2012. Series de precios agropecuarios. En <http://www.aacrea. org.ar/index.php/software/141-serie-de-preciosagropecuarios.htm> Consultada el 20/12/ 2012.

AOAC (Association of Official Analytical Chemists), 1990. Official methods of analysis: First supplement. 15th. Ed. AOAC, Arlington, VA.

Arzadun, M.J.; J.I. Arroquy, H.E. Laborde and R.E. Brevedan. 2003. Grazing pressure on beef and grain production of dual-purpose wheat in Argentina. Agron. J. 95:1157-1162.

Bainotti, C.; M. Arzadun, L. Lazaro, H. Laborde, R. Bandera, J. Lopez, A. Bolleta, E. Grassi, B. Masiero y C. Ghida Daza. 2008. Evaluación de cultivares de trigo doble propósito durante la campaña 2006/07. Información para Extensión Nro. 118 INTA EEA Marcos Juárez, 10 pp.

Denda, S.; M. Arzadun, H. Arelovich, H. Laborde y M. Torrea, 2004. Influencia de la fertilización nitrogenada sobre la calidad y composición química del forraje de trigo. Rev Arg Prod Animal 24 (s1):82-83.

Dobermann, A., 2007. Nutrient use efficiency - measurement and management. IFA International workshop on fertilizer best management practices. pp 1-28.

Fernando, G.W.E. and Carter, O.G. 1970. The effect of level of nitrogen fertilizer applied to forage oat on the grazing behavior of dairy cattle. Proceedings $\mathrm{XI}^{\circ}$ Int Grassland Congr. Brisbane, Australia. pp 853 
Freddi, J.; M. Arzadún, A. Pissani and P. Sastre, 1997. Oat grazing: forage composition and supplementation response. XVIII International Grassland Congress, Winnipeg-Saskatoon, Canada. Proceedings 2:155-156.

Freddi, J.; M. Arzadun, O. Sánchez y C. Ibarra, 2003. Producción de forraje y carbohidratos soluble de cinco verdeos de invierno. Rev Arg Prod Animal 23 (s1):202204.

Goering H. and P. Van Soest,1970. Forage fiber analyses (apparatus, reagents, procedures and some applications). USDA - ARS. Washington DC. Agricultural Handbook 379. 20 pp.

Golik, S.; H. Chidichimo, D. Pérez y L. Pane, 2003. Acumulación, removilización, absorción postantesis y eficiencia de utilización de nitrógeno en trigo bajo diferentes labranzas y fertilizaciones. Pesq Agropec. Bras. 38:619-626.

Hernández, O., 1969. Efecto de la época de siembra e intensidad del pastoreo sobre el rendimiento en grano de trigo doble propósito. Revista de Investigación Agrícola 6:155-165.

Huggins, D. and W. Pan, 1993. Nitrogen efficiency component analysis: An evaluation of cropping system differences in productivity. Agron. J. 85:898-905.

Johnston, A. and D. Fowler, 1991. No-till winter wheat production: Response to spring applied nitrogen fertilizer form and placement. Agron. J. 83:722-728.

Keeney, D. and D. Nelson, 1982. Nitrogen-inorganic forms. In A.L. Page et al (ed) Methods of soil análisis. Part 2. 2nd ed. SSSA Book Ser. 5. SSSA, Madison, WI. pp. 643-698.

Laurent, G., M. Lázzari y R. Victoria, 1996. Balance del nitrógeno del fertilizante aplicado al trigo en dos épocas diferentes. Ciencia del Suelo 14:7-11.

Lázzari, M., 1992. Estudios de balance de nitrógeno usando ${ }^{15} \mathrm{~N}$. Rev Facultad de Agronomía. UBA. 13:127-132.

Loewy, T., 1990. Fertilización nitrogenada del trigo en el sudoeste bonaerense. I. Respuesta física y diagnostico. Ciencia del Suelo 8:47-56.

Loewy, T., 2004. Fraccionamiento del nitrógeno y fertilización foliar en trigo. Actas del XIX ${ }^{\circ}$ Congreso Argentino de la Ciencia del Suelo. Paraná (Entre Ríos), 22-25 de junio de 2004. En CD Rom.

Mackown, C. and B. Carver, 2005. Fall forage biomass and nitrogen composition of winter wheat populations selected from grain-only and dual-purpose environments. Crop Science 45:322-328.

N.R.C. (National Research Council) 1980. Necesidades nutritivas del ganado vacuno de carne. Editorial Hemisferio Sur. Buenos Aires. 80 pp.

Peralta, N.; P. Abbate y A. Marino, 2011. Efecto del regimen de defoliación sobre la producción de grano de un trigo doble propósito. Agriscientia 28:1-11.

Pordomingo, A.; N. Juan y A. Pordomingo, 2007. Relación entre el aumento de peso de novillos sobre verdeos de invierno y parámetros de calidad del verdeo (comunicación). (Resumen NA 55).30 Congreso Argentina de Producción Animal. Revista Argentina de Producción Animal 27 (1):83-84.

Redmon, L.; G. Horn, E. Krenzer and D. Bernardo, 1995. A review of livestock grazing and wheat grain yield: Boom or bust? Agron. J. 87:137-147.

Raun, W. and R. Westerman, 1991. Nitrate-N and Phosphate-P concentrations in winter wheat at varying growth stages. J. Plant. Nutr. 14:267-281.

Raun, W. and G. Johnson, 1999. Improving Nitrogen Use Efficiency for cereal production. Review and interpretation. Agron J. 91:357-363.

Raun, W.; J. Solie, G., Johnson, M. Stone, R. Mullen, K. Freeman, W. Thomason and E. Lukina, 2002. Improving nitrogen use efficiency in cereal grain production with optical sensing and variable rate application. Agron. J. 94:815-820.

Sowers, K.; W. Pan, B. Miller and J. Smith, 1994. Nitrogen use efficiency of split applications in soft white winter wheat. Agron J. 86:942-948.

Thomas, A., 1977. An automated procedure for the determination of soluble carbohydrates in herbage. J. Sci. Food Agric. 28: 639-642.

Thomason, W.; W. Raun and G. Johnson, 2000. Winter wheat fertilizer nitrogen use efficiency in grain and forage production systems. Journal of Plant Nutrition, 23:1505-1516. 